\title{
1 Hormone Levels Are Related to Altered Functional Connectivity in
}

\section{Prolactinomas}

3 Running head: Hormone-Connectivity Relationship in Prolactinomas

4 Shun Yao, MD, $\mathrm{PhD}^{1,2,3}$, Chenglong Cao, $\mathrm{MD}^{4,2}$, Pan Lin, $\mathrm{PhD}^{5,6}$, Parikshit Juvekar, MBBS 3 ,

5 Ru-Yuan Zhang, $\mathrm{PhD}^{7}$, Matthew Vera, M.S. ${ }^{3}$, Ailiang Zeng, MD ${ }^{8,9}$, Alexandra J. Golby, MD,

$6 \mathrm{PhD}^{3}$, Guozheng $\mathrm{Xu}, \mathrm{MD}, \mathrm{PhD}^{2}$, Yanmei Tie, $\mathrm{PhD}^{3 *}$, Jian Song, $\mathrm{MD}, \mathrm{PhD}^{2 *}$

$7{ }^{1}$ Department of Neurosurgery and Pituitary Tumor Center, The First Affiliated Hospital, Sun Yat-sen

8 University, Guangzhou, China

$9{ }^{2}$ Department of Neurosurgery, General Hospital of Chinese PLA Central Theater Command, Wuhan,

10 China

$11{ }^{3}$ Department of Neurosurgery, Brigham and Women's Hospital, Harvard Medical School, MA, United

12 States

$13 \quad{ }^{4}$ Wuhan School of Clinical Medicine, Southern Medical University, Guangzhou, China

$14{ }^{5}$ Department of Psychology, Hunan Normal University, Changsha, China

$15{ }^{6}$ Cognitive and Human Behavior Key Laboratory of Hunan Province, Hunan Normal University,

16 Changsha, China

$17{ }^{7}$ Center for Magnetic Resonance Research, Department of Neuroscience, University of Minnesota at Twin

18 Cities, MN, United States

$19{ }^{8}$ Department of Neurosurgery, The First Affiliated Hospital of Nanjing Medical University, Nanjing,

20 China

21 'Department of Neurology, Brigham and Women's Hospital, Harvard Medical School, Boston, MA,

22 United States

\section{Corresponding Author:}

\section{Jian Song, MD., Ph.D}

25 Department of Neurosurgery, General Hospital of Chinese PLA Central Theater Command, 627 Wuluo 26 Road, Wuhan 430070, China. E-mail: docsongjian@yahoo.com TEL: (+85)18502760505

\section{Yanmei Tie, Ph.D}

Department of Neurosurgery, Brigham and Women's Hospital, Harvard Medical School, Hale Building for Transformative Medicine 8016G, 60 Fenwood Road, Boston, MA 02115, U.S. E-mail: ytie@bwh.harvard.edu TEL: (+1)617-732-8249

\section{Word counts of the main document: 1246words}




\section{ABSTRACT}

35 Background and Objective: Prolactinomas may cause drastic hormone fluctuations throughout

36 the body. It is not fully understood how endogenous hormone disorders such as prolactinomas

37 reshape the patient's brain. By employing the resting-state functional magnetic resonance imaging 38 technique, we aimed to investigate the whole-brain functional connectivity (FC) and its 39 relationship with hormone levels in patients with prolactinomas.

40 Methods: Using whole-brain and seed-based functional connectivity analyses, we compared FC 41 metrics between 33 prolactinoma patients and 31 healthy controls matched with age, sex, and

42 handedness. Then we performed partial correlation analysis to examine the relationship between $43 \quad$ FC metrics and hormone levels.

44 Results: Compared to healthy controls, we found that prolactinoma patients showed significantly 45 increased thalamocortical (visual cortex) and cerebellar-cerebral connectivity. In addition, 46 endogenous hormone levels were positively correlated with the increased FC, and the hormone$47 \quad$ FC relationships showed sex difference in prolactinoma patients.

48 Conclusions: Our findings are the first to reveal the altered FC patterns and sex-dependent 49 hormone-FC relationships in prolactinoma patients, indicating the important role of hormone 50 levels in the neural mechanism of brain reorganization and hyperactive intrinsic connections in 51 prolactinomas. INTRODUCTION

54 Prolactinomas are characterized by a dramatic surge of prolactin that suppresses the secretion of sex steroid hormones. Evidence has been found that sex steroids can affect the structural and 56 functional organization of the brain. We have previously investigated the influence of endogenous 57 hormones on brain gray matter and neurocognition in prolactinoma patients. ${ }^{1}$ However, functional connections in prolactinoma patients have scarcely been explored. Recently, the resting-state 59 functional magnetic imaging (rsfMRI) has become a useful tool to characterize the brain's 60 functional connectivity (FC) pattern that is considered a potential metric reflecting the temporal

61 dependence of neuronal activation patterns of spatially separated brain areas. ${ }^{2}$

62 The aim of the current study was to determine the functional connectivity patterns in 63 prolactinomas. We hypothesized that prolactinoma patients would show abnormal functional 64 connectivity patterns related to the increased endogenous hormone levels. 


\section{METHODS}

\section{Study Population}

68 Thirty-three prolactinoma patients and 31 healthy controls participated in this study. All patients were recruited in the Department of Neurosurgery during diagnostic hospitalization. The inclusion and exclusion criteria were described in our previous work ${ }^{1}$.

All procedures were under the Declaration of Helsinki and approved by the Ethical Committee of General Hospital of Chinese PLA Central Theater Command (Approved ID: [2017] 024-1). The study protocol was fully explained, and written informed consent was acquired from all

74 participants.

\section{Hormone Assays and Visual Assessment}

77 The measurement of hormone levels in serum was described in our previous work ${ }^{1}$. All patients

78 routinely underwent complete ophthalmologic examinations at admission. The E chart was used

79 to measure the best-corrected visual acuity. The visual field parameter was obtained using the 80 standardized, automated perimetry (Octopus 900 Perimetry, Switzerland).

\section{MRI Data Acquisition}

83 The MRI data were acquired with a 1.5 Tesla scanner (GE EXCITE, Milwaukee, USA) using an

84 8-channel head coil. The structural MRI acquisition was described in our previous study ${ }^{1}$. Blood 85 oxygen level dependent (BOLD) fMRI data were acquired using echo-planar images (TR/TE $862000 / 30 \mathrm{~ms}, 4.0 \mathrm{~mm}$ thickness, $0.5 \mathrm{~mm}$ gap, 33 axial slices, matrix size of $64 \times 64$, and $7 \mathrm{~min}$ ).

87 During rsfMRI, all participants were instructed to keep their eyes open with a central fixation condition.

\section{fMRI Preprocessing}

91 fMRI data were preprocessed using the CONN Toolbox (v.18.a;

92 http://www.nitrc.org/projects/conn) $\quad$ based $\quad$ on $\quad$ SPM12

93 (http:/www.fil.ion.ucl.ac.uk/spm/software/spm12) in MATLAB R2018a (MathWorks, Inc., MA,

94 USA). Standard preprocessing steps of rsfMRI included realignment, slice-timing correction, 95 functional outlier detection, normalization, Gaussian spatial smoothing (6 mm FWHM), bandpass 
filtering (0.008-0.09 Hz), and cleaning of motion and physiological noise using the CompCor

97 method.

\section{Whole-brain Functional Connectivity Analysis}

100 For the whole-brain FC analyses, 132 regions of interest (ROIs) were defined using the brain 101 parcellation implemented in the CONN toolbox. The mean time series of each ROI was extracted 102 to construct the ROI-to-ROI correlation matrices, which were then converted to $\mathrm{z}$ scores using 103 Fisher's z-transformation. To correct multiple comparisons between groups, a seed-level false 104 discovery rate (FDR) method with the significant threshold of $p<0.05$ was performed.

Statistical Analysis

107 Group differences were compared using two-sample student's t-test or Pearson's Chi-Squared test, 108 as appropriate. The partial correlation analysis was performed to determine the relationship 109 between FC metrics and hormone levels controlling the factors of age, tumor volume, and disease 110 history. Significance was set at $\mathrm{p}<0.05$ (two-tailed). Statistical analyses were performed using 111 JASP version 0.10 .2 (https://jasp-stats.org/).

RESULTS

\section{Clinical Characteristics}

115 There were no differences between prolactinoma patients and healthy control in age $(p=0.266)$ 116 and sex $(p=0.477)$. The other clinical characteristics were described in Table 1.

\section{Altered Whole-brain FC Patterns}

119 Compared to healthy controls, prolactinomas showed increased FC between the left thalamus and 120 visual cortex/association areas [bilateral lingual gyrus (LG), right intra-calcarine cortex (ICC), 121 right supra-calcarine cortex (SCC), right cuneal cortex, and bilateral lateral occipital cortex (LOC)] 122 and right cerebellum (Cereb), the right cerebellum and cerebral cortex [precuneus, posterior 123 cingulate gyrus (PC), bilateral temporal fusiform cortex (TFusC)], and the left supplemental motor area (SMA) and right lingual gyrus (Fig. 1). In addition, the increased FC of the thalamus and

125 visual cortex were more prominent in patients with visual field impairments (VFI) compared to 126 patients without VFI (Supplementary Table 1). 


\section{Relationship between Hormone Levels and FC Metrics}

In prolactinomas, females showed a significantly positive correlation between the prolactin level and the FC of left thalamus and right lingual gyrus. Males showed a positive correlation between the testosterone level and the FC of right cerebellum and left fusiform gyrus as well as the correlation between the LH level and the FC of left SMA and right lingual gyrus (Fig. 2).

\section{DISCUSSION}

To our knowledge, this study is the first to demonstrate the altered whole-brain FC patterns in prolactinomas and their relationships with endogenous hormone levels.

137 Our first notable finding is the illustration of increased functional connectivity between the left 138 thalamus and visual cortex/association areas (lingual gyrus, ICC, SCC, cuneal cortex, and LOC). 139 The thalamus is both the primary integrator and the gateway of sensory input to the cerebral cortex. Visual cortex/association areas mainly receive input of visual signals coming from the retina via the lateral geniculate nucleus of the thalamus. Considering the different visual-related FC patterns between patients with VFI and without VFI, these hyperactive intrinsic connections may illustrate compensatory activity within the visual system, which is consistent with a previous study. ${ }^{3}$

145 the right cerebellum to the default mode network $(\mathrm{DMN})^{4}$ (posterior cingulate cortex (PCC)/precuneus), and to bilateral TFusC. The PCC/precuneus plays a pivotal role in controlling the state of arousal and maintaining attention. ${ }^{4}$ TFusC mediates a variety of higher cognitive and

148 emotional functions that relate to the processing of face/object perception. ${ }^{5}$ The cerebellum is also prominently activated in control of negative emotional processing and occurred concomitantly with the mirror neuron system, including TFusC and precuneus. ${ }^{6}$ Thus, there might exist functional connections between the cerebellum and DMN/TFusC. In addition, the thalamus plays a critical role in modulating the communication within cortico-cerebello-cortical parallel closed loops that involve DMN, salience, attention, and sensorimotor networks. ${ }^{7}$ Therefore, the increased FC of

154 cerebellum and fusiform/DMN/thalamus may contribute to the hyperactive intrinsic connections 155 serving dysfunctional cognitive or emotional processing in prolactinomas.

156 Hormones affect brain development, organization, and plasticity. ${ }^{8}$ The prolactin receptor is 157 distributed broadly in the brain and can mediate the regulation of neuronal excitability, 
158

159

160

161

162

163

164

165

166

167

168

169

170

171

172

173

174

175

176

177

178

179

180

181

182

183

184

185

186

187

neurotransmission, and channels in the nervous system. ${ }^{9}$ Evidence has emerged supporting the critical role of tuberoinfundibular dopamine neurons in the remarkable plasticity and the necessity of their function during lactation. ${ }^{10}$ However, owing to the anti-correlation of prolactin and dopamine, the underlying mechanism of how prolactin influences brain reorganization is unknown. LH and testosterone may contribute to brain reorganization by modulating neural plasticity through routes engaging actin sytoskeleton ${ }^{11}$ and the growth of white matter in the brain. ${ }^{12}$ Thus, our findings of the relationships between hormone levels and the altered FC may illustrate the potential influence of endogenous hormones in brain reorganization in prolactinoma patients who usually display a cascade of hormone disorders.

However, several limitations should be addressed. First, the mass effect of the bulky tumor on surrounding structures was challenging to be completely ruled out even the tumor size was adjusted during the data analysis. Second, diffusion tensor imaging could be employed to assess the structural integrity of the visual pathway in future work. Third, the relationship between hormone levels and the altered FC in males might have been biased due to the relatively small sample size.

Overall, our findings provide new insights into the altered FC patterns and the important role of endogenous hormones in the neural mechanism of brain reorganization in prolactinomas.

\section{FUNDING}

This work is supported by the funding from the National Science Foundation of China (NSFC) through grants: No.81571049 and No.81870863 and the National Institutes of Health (NIH) through Grants: R21CA198740, P41EB015898, P41RR019703, and R25CA089017. Shun Yao received the scholarship from the State Scholarship Fund, China Scholarship Council (201808440461).

\section{AUTHOR CONTRIBUTIONS}

G.X. and J.S. provided the major research funding. S.Y., A.J.G., G.X., Y.T., and J.S. contributed to the conception and design of the study. S.Y. collected data, performed the data analysis, and drafted the manuscript. P.L. and C.C. did the brain tumor segmentation and J.S. reviewed all lesion masks (all of them were blind to the clinical data). P.L., P.J., W.V., A.Z., A.J.G., and R.Z 
participated in the fMRI data preprocessing and edited the manuscript. Critically revising the article: all authors. Approved the final version of the manuscript on behalf of all authors.

\section{DISCLOSURE}

192 The authors declare no conflicts of interest.

193

194

195

196

197

198

199

200

201

202

203

204

205

206

207

208

209

210

211

212

213

214

215

216

217

218

219

220

221

\section{REFERENCES:}

1. Yao S, Song J, Gao J, et al. Cognitive Function and Serum Hormone Levels Are Associated with Gray Matter Volume Decline in Female Patients with Prolactinomas. Front Neurol [online serial]. 2018;8. Accessed at: https://www.frontiersin.org/articles/10.3389/fneur.2017.00742/full\#. Accessed February 2, 2018.

2. van de Ven VG, Formisano E, Prvulovic D, Roeder CH, Linden DEJ. Functional connectivity as revealed by spatial independent component analysis of fMRI measurements during rest. Hum Brain Mapp. 2004;22:165-178.

3. Qian H, Wang X, Wang Z, Wang Z, Liu P. Altered Vision-Related Resting-State Activity in Pituitary Adenoma Patients with Visual Damage. PLoS ONE. 2016;11:e0160119.

4. Fransson P, Marrelec G. The precuneus/posterior cingulate cortex plays a pivotal role in the default mode network: Evidence from a partial correlation network analysis. NeuroImage. 2008;42:1178-1184.

5. Weiner KS, Zilles K. The anatomical and functional specialization of the fusiform gyrus. Neuropsychologia. 2016;83:48-62.

6. Schraa-Tam CKL, Rietdijk WJR, Verbeke WJMI, et al. fMRI Activities in the Emotional Cerebellum: A Preference for Negative Stimuli and Goal-Directed Behavior. Cerebellum. 2012;11:233-245.

7. Habas C, Manto M, Cabaraux P. The Cerebellar Thalamus. Cerebellum. 2019;18:635-648.

8. Garcia-Segura LM, Melcangi RC. Steroids and glial cell function. Glia. 2006;54:485-498.

9. Patil MJ, Henry MA, Akopian AN. Prolactin receptor in regulation of neuronal excitability and channels. Channels (Austin). 2014;8:193-202.

10. Barth C, Villringer A, Sacher J. Sex hormones affect neurotransmitters and shape the adult female brain during hormonal transition periods. Front Neurosci [online serial]. 2015;9. Accessed at: https://www.frontiersin.org/articles/10.3389/fnins.2015.00037/full. Accessed February 13, 2019. 
222 11. Edwards BS, Clay CM, Ellsworth BS, Navratil AM. Functional Role of Gonadotrope

Plasticity and Network Organization. Front Endocrinol [online serial]. 2017;8. Accessed at: https://www.frontiersin.org/articles/10.3389/fendo.2017.00223/full. Accessed July 13, 2019.

226

12. Perrin JS, Hervé P-Y, Leonard G, et al. Growth of White Matter in the Adolescent Brain: 
Tables and Figure Legends

230 Table 1 Clinical Features Comparison between Prolactinomas and Healthy Controls

\begin{tabular}{lccc}
\hline Clinical categories & $\begin{array}{c}\text { Prolactinomas } \\
(\mathrm{n}=33)\end{array}$ & $\begin{array}{c}\text { Healthy controls } \\
(\mathrm{n}=31)\end{array}$ & P value \\
\hline Age (years) & $43.76 \pm 10.87$ & $46.74 \pm 10.38$ & $0.266^{\mathrm{a}}$ \\
Gender (F/M) & $22 / 11$ & $18 / 13$ & $0.477^{\mathrm{b}}$ \\
History of Disease & $12.0(6.0-24.0)$ & $\mathrm{N} / \mathrm{A}$ & \\
(months), median (IQR) & & & \\
Volume of Pituitary & $4.4(1.7-9.8)$ & $\mathrm{N} / \mathrm{A}$ & \\
Tumor (cc), median (IQR) & & & \\
Clinical Symptoms & & $\mathrm{N} / \mathrm{A}$ & \\
Headache & $24.3 \%(8 / 33)$ & $\mathrm{N} / \mathrm{A}$ & \\
Visual impairments & $45.5 \%(15 / 33)$ & $\mathrm{N} / \mathrm{A}$ & \\
Sexual dysfunction & $48.5 \%(16 / 33)$ & & \\
Hormone levels & & $\mathrm{N} / \mathrm{A}$ & \\
Estradiol (pg/ml) & $40.5 \pm 79.31$ & $\mathrm{~N} / \mathrm{A}$ & \\
FSH (mIU/ml) & $8.31 \pm 9.08$ & $\mathrm{~N} / \mathrm{A}$ & $\mathrm{N} / \mathrm{A}$ \\
LH (mIU/ml) & $3.65 \pm 3.39$ & $\mathrm{~N} / \mathrm{A}$ \\
Progesterone (ng/ml) & $1.20 \pm 3.76$ & $\mathrm{~N} / \mathrm{A}$ \\
Prolactin (ng/ml) & $190.3 \pm 177.6$ & $\mathrm{~N} / \mathrm{A}$ & \\
Testosterone (ng/ml) & $0.92 \pm 1.37$ & $2.96 \pm 2.57$ & \\
TSH (uIU/ml) & $291.9 \pm 174.4$ & & \\
Cortisol (nmol/l) & $0.57 \pm 0.93$ & & \\
GH (ng/ml) & & & \\
\hline
\end{tabular}

231 a two-sample student's t-test

232 b Pearson Chi-Squared test of Fisher's exact test

233 F, female; M, male; IQR, interquartile range; cc, cubic centimeter; FSH, follicle-stimulating

234 hormone; LH, luteinizing hormone; THS, thyroid-stimulating hormone; GH, growth hormone. 
237 Figure 1. ROI-to-ROI analyses of the resting-state functional connectivity across the whole238 brain. Compared to healthy controls, significantly increased functional connectivity was only

239 shown in patients with prolactinomas. The false discovery rate (FDR) method was used to

240 perform the seed-level two-sided correction at a significant level of $p<0.05$. The nodes rendered

241 by the red color indicated more connections to other nodes where the nodes rendered by the

242 green color indicated less connections to other nodes, and the yellow-rendered nodes indicate

243 mediate number connections in the whole connectivity pattern.

244 Abbreviations: Cereb, Cerebellum; pTFusC, temporal fusiform cortex, posterior division; LG,

245 lingual gyrus; sLOC, lateral occipital cortex, superior division; ICC, intra-calcarine cortex; SCC,

246 supra-calcarine cortex; PC, cingulate gyrus, posterior division; SMA, supplemental motor area.

247
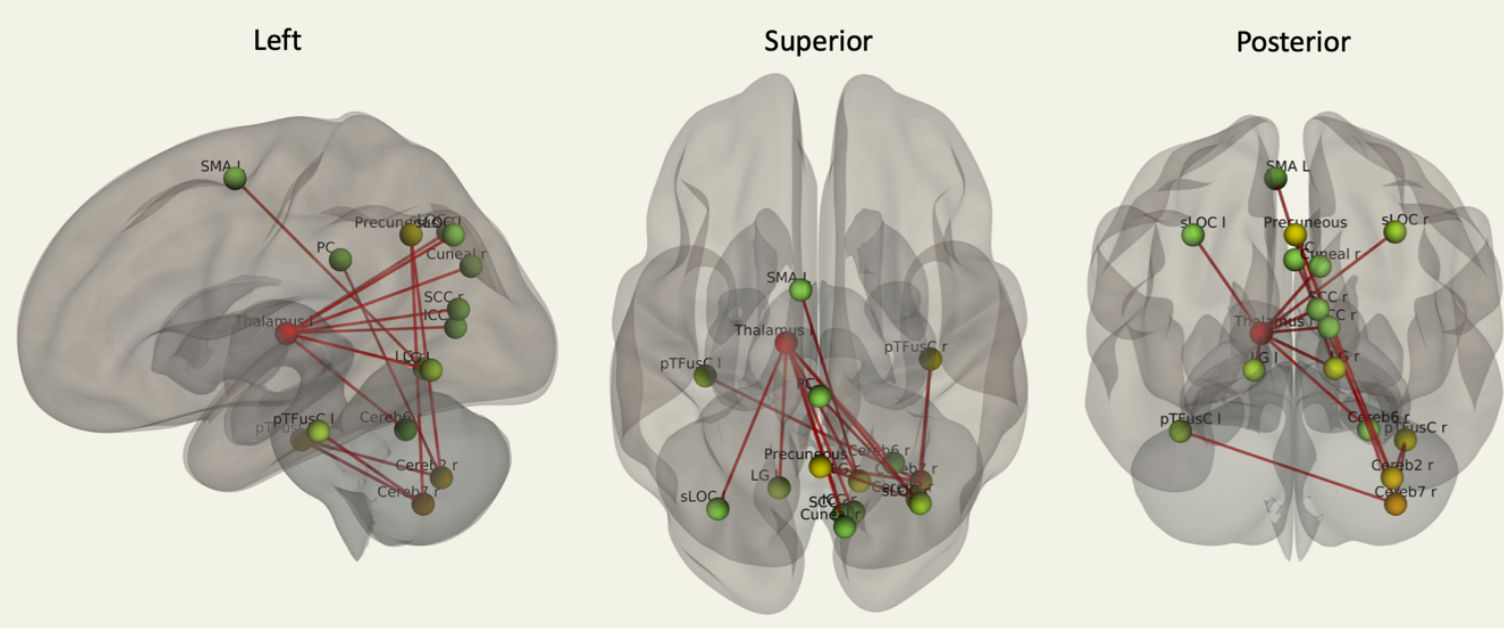
249 Figure 2. Partial correlations analyses between endogenous hormone levels and altered

250 functional connectivity metrics. (A) the PRL level in serum is positively correlated with the FC

251 of the left thalamus and right LG. (B) the T level in serum is positively correlated with the FC of

252 the right cerebellum and left TFusC. (C) the LH level in serum is positively correlated with the

253 FC of the left SMA and right LG. The shading area indicates the 95\% confidence interval of a

254 correlation line.

255 Abbreviations: PRL, prolactin; T, testosterone; LH, luteinizing hormone; LG, lingual gyrus;

256 TFusC, temporal fusiform cortex; SMA, supplemental motor area.

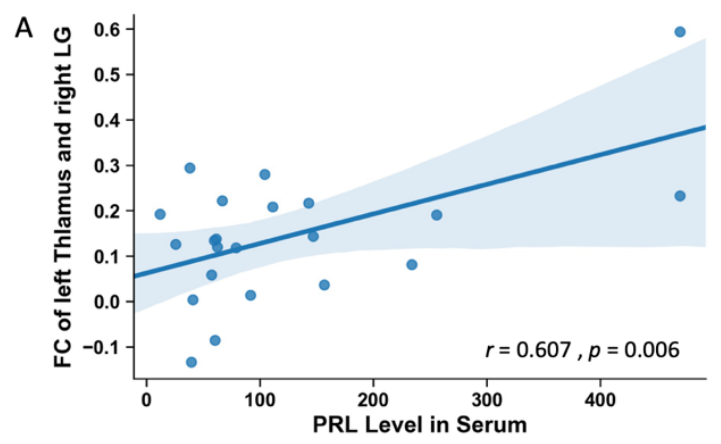

B

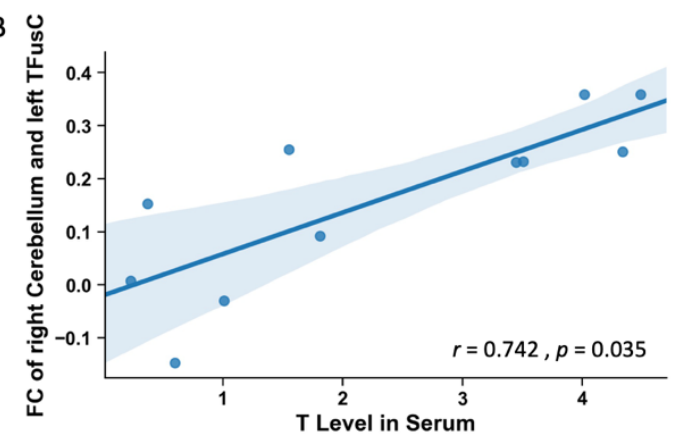

C

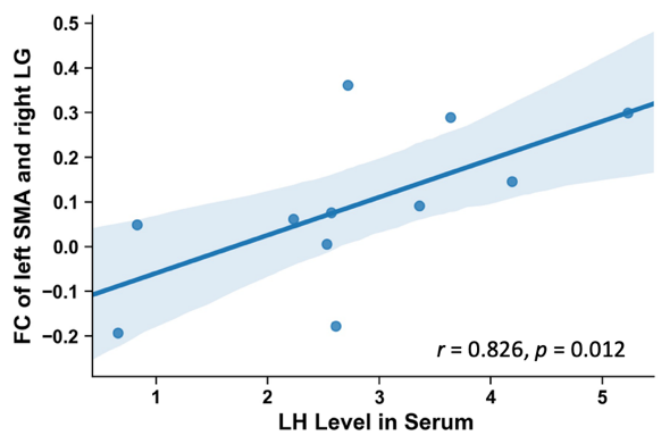




\section{Left}

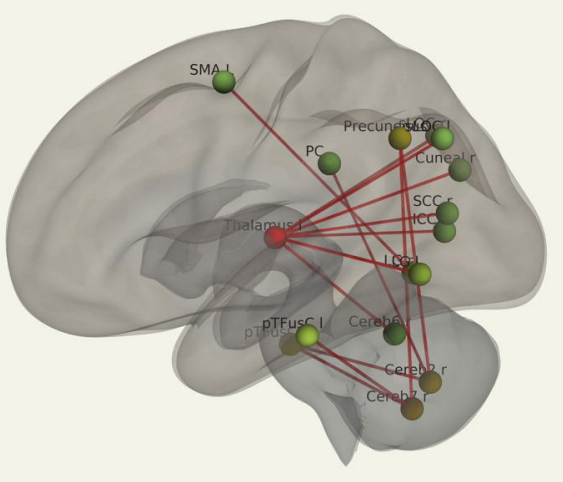

Superior

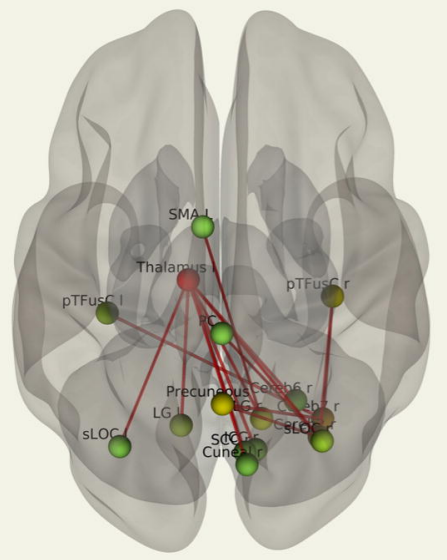

\section{Posterior}

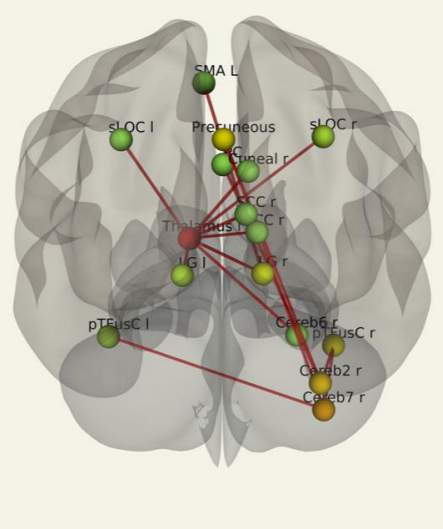



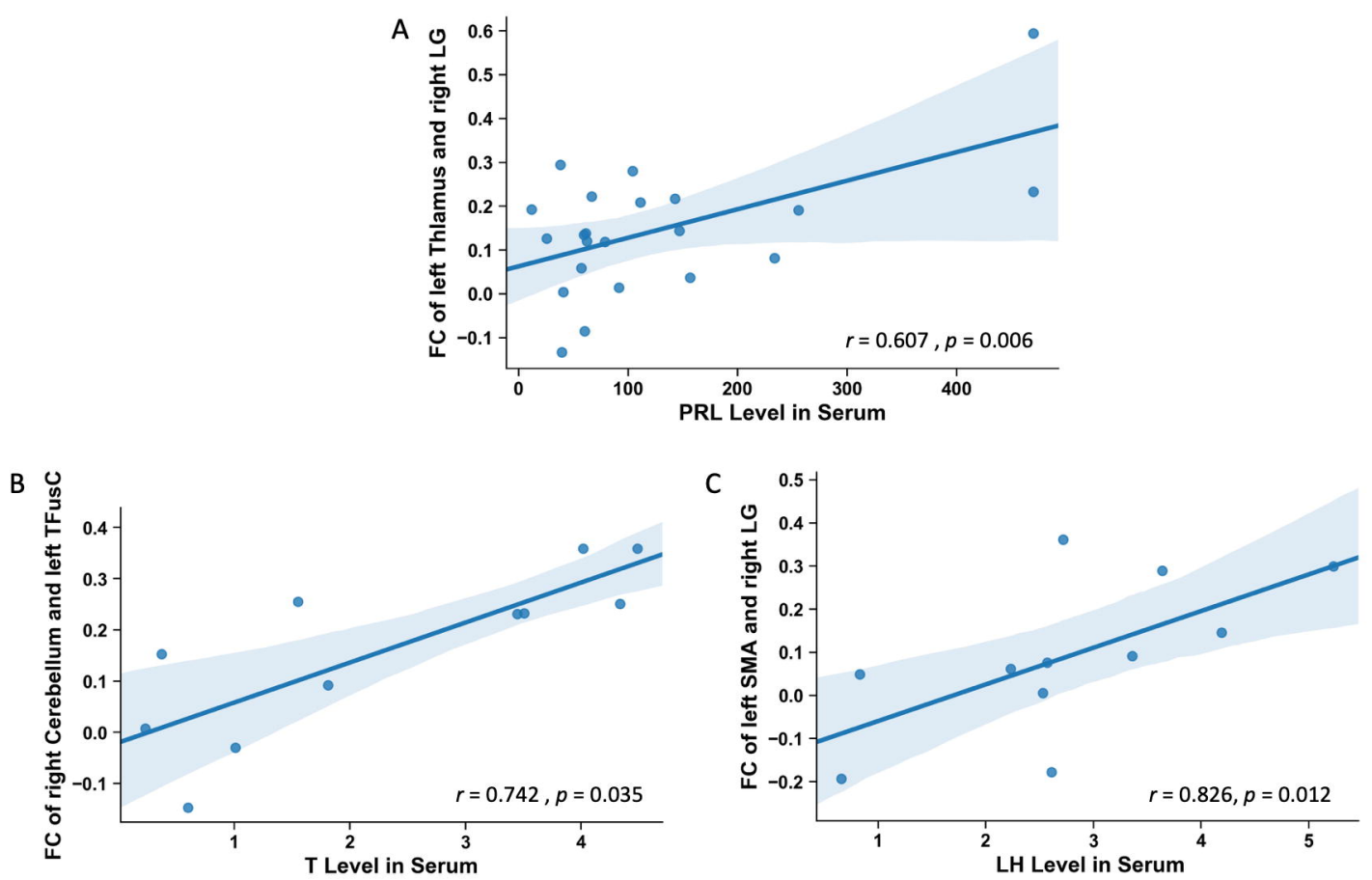